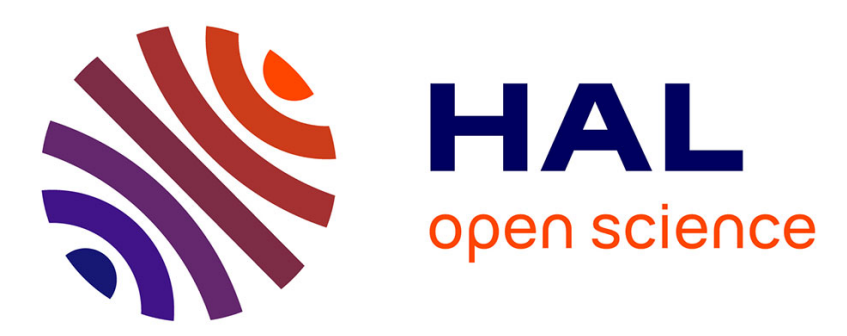

\title{
Argumentation et séduction: Étude de quelques connecteurs argumentatifs dans un dialogue de Crébillon fils
}

Geneviève Salvan

\section{- To cite this version:}

Geneviève Salvan. Argumentation et séduction : Étude de quelques connecteurs argumentatifs dans un dialogue de Crébillon fils. L'information grammaticale, 1996, 68, pp.25-30. 10.3406/igram.1996.3018 . hal-01182222

\author{
HAL Id: hal-01182222 \\ https://hal.science/hal-01182222
}

Submitted on 31 Jul 2015

HAL is a multi-disciplinary open access archive for the deposit and dissemination of scientific research documents, whether they are published or not. The documents may come from teaching and research institutions in France or abroad, or from public or private research centers.
L'archive ouverte pluridisciplinaire HAL, est destinée au dépôt et à la diffusion de documents scientifiques de niveau recherche, publiés ou non, émanant des établissements d'enseignement et de recherche français ou étrangers, des laboratoires publics ou privés. 


\section{Argumentation et séduction : Étude de quelques connecteurs argumentatifs dans un dialogue de Crébillon fils}

In: L'Information Grammaticale, N. 68, 1996. pp. 25-29.

Citer ce document / Cite this document :

Salvan Geneviève. Argumentation et séduction : Étude de quelques connecteurs argumentatifs dans un dialogue de Crébillon fils. In: L'Information Grammaticale, N. 68, 1996. pp. 25-29.

doi : 10.3406/igram.1996.3018

http://www.persee.fr/web/revues/home/prescript/article/igram_0222-9838_1996_num_68_1_3018 


\section{ARGUMENTATION ET SÉDUCTION Étude de quelques connecteurs argumentatifs dans un dialogue de Crébillon fils}

\section{Geneviève SALVAN}

Toute l'œuvre de Crébillon - et notamment Les Égarements - est envahie par les échanges verbaux entre personnages, échanges dont le but est souvent - voire toujours - de séduire l'autre. Ces conversations sont un formidable objet d'étude pour dégager les " principes " des mécanismes argumentatifs mis en œuvre dans et par le discours, mais également pour réfléchir au problème de l'interprétation des énoncés et de son " rendement " stylistique.

Dans le vaste champ des études des ressources argumentatives du discours, nous nous intéresserons plus particulièrement aux connecteurs argumentatifs - spécialement mais - sortes de chevilles ouvrières de l'argumentation et révélateurs des enjeux profonds de l'interaction. Les occurrences analysées se situent dans la " première " conversation entre Meilcour et Lursay (nous savons que ce n'est pas la toute première dans l'ordre de la mimesis mais seulement dans l'ordre de la diegesis(1)). Madame de Lursay est une amie de la mère de Meilcour et depuis six mois sert de "partenaire discursif" au jeune homme, qui découvre les affres des premiers désirs et les tourments de l'amour encore sans objet. Une conversation s'installe entre eux à propos d'une pièce de théâtre vue la veille: dialogue célèbre qui a été déjà bien étudié(2), et dont nous ne rendrons pas compte en général. Nous donnons juste le relevé des occurrences:

\section{RELEVÉ DES OCCURRENCES}

"L'intrigue, dit-elle, ne m'en paraît pas neuve, mais (1) j'en aime assez les détails: elle est noblement écrite, et les sentiments y sont bien développés. N'en pensez-vous pas comme moi? Je ne me pique pas d'être connaisseur, répondis-je. En général elle m'a plu; mais (2) j'aurais peine à bien parler de ses beautés et de ses défauts." (GF, 1985: 77)

" (...) et vous cesseriez de sentir du goût pour celle [la femme] qui vous en aurait inspiré le plus, dans l'instant qu'elle vous offritait une conquête aisée. Cela n'est pas raisonnable, dis-je;

1. «Dans quelques conversations que nous avions eues ensemble sur l'amour, elle s'était instruite de mon caractère, et des raisons qui pouvaient me faire redouter l'aveu d'une passion que j'aurais conçue." (GF: 74-75)

2. Voir les articles suivants: Anne Giard, "La parole soufflée ", Littérature $n^{\circ}$ 31, 1978: 64-76, et Anne-Marie Paillet-Guth, "Marivaux, Crébillon et la mauvaise foi : les détours d'une déclaration d'amour ", Revue Marivaux $n^{\circ} 1$, 1990: 53-64. Voir également Carole Dornier, "Les Égarements: La manipulation d'un jeune homme expérimenté ", dans L'énoncé général dans l'œuvre de Crébillon fils troisième partie, chapitre II, thèse de doctorat, Caen, 1990. et l'on doit à ce qu'il me semble, plus de reconnaissance à quelqu'un qui vous épargne des tourments... Sans doute, interrompit-elle; mais (3) vous pensez mal pour votre intérêt et pour le nôtre. " (78)

"Les déclarations les plus élégantes ne sont pas toujours, répondit-elle, les mieux reçues. On s'amuse de l'esprit d'un amant, mais ce n'est pas lui qui persuade ; son trouble, la difficulté qu'il trouve à s'exprimer, le désordre de ses discours, voilà ce qui le rend à craindre. Mais (4), Madame, lui demandai-je, cette preuve, qui en effet me paraît incontestable, persuade-telle toujours?" (79)

" (...) il est plus avantageux, même plus raisonnable, de parler que de s'obstiner à se taire. Vous risquez de perdre par le silence le plaisir de vous savoir aimé; et si l'on ne peut vous répondre comme vous le voudriez, vous vous guérissez d'une passion inutile qui ne fera jamais que votre malheur. Mais (5), ajoutat-elle, je remarque que depuis longtemps vous me parlez sur ce sujet, et si je ne me trompe, une déclaration ne vous paraît embarrassante que parce que vous en avez une à faire. " (79)

\section{A. MISE AU POINT SUR LA NOTION DE CONNECTEUR}

\section{Définition et perspectives}

Les connecteurs sont des morphèmes grammaticaux qui ont une fonction pragmatique, ils donnent des instructions pour linterprétation d'un énoncé. De façon traditionnelle, on distingue deux fonctions des connecteurs - dont une seule nous retiendra - celle de structurant du texte (au niveau de la progression et de l'enchaînement des propositions, et ce dans une perspective narratologique large) (3) et un rôle d'instructeur de la cohérence pragmatique d'un énoncé. Ce qui nous importe, c'est de voir comment les connecteurs participent à une argumentation, c'est-à-dire à une " action complexe finalisée, un enchaînement structuré d'arguments liés par une stratégie globale qui vise à faire adhérer l'auditoire à la thèse défendue par l'énonciateur (4). "Si, dans notre texte, il n'y a pas de thèse explicitement dévoilée, l'action de la Marquise est néanmoins de faire " rentrer " Meilcour dans un univers de sens donné, de modifier l'état de ses

3. Voir les travaux de J.-M. Adam, et particulièrement: " Des mots au discours: l'exemple des principaux connecteurs ", Pratiques $n^{\circ} 43$, octobre 1984: 107-122 et Les textes : types et prototypes, Paris, Nathan Université, 1992.

4. D. Maingueneau, L'Analyse du discours, Introduction aux lectures de l'archive, 1991: 228. 
convictions et de lui donner une compétence rhétorico-pragmatique (5) pour lui permettre de tenir sa place - de séducteur-dans le monde.

Plusieurs théories des connecteurs ont été formulées (6), en France autour d'Oswald Ducrot et de J.-C. Anscombre, en Suisse autour d'Eddy Roulet (perspective conversationnelle) et également d'A. Berrendonner, enfin dans le cadre de la théorie de le pertinence fondée par $D$. Sperber et $D$. Wilson, avec J. Mœschler et J.-M. Luscher. La théorie d'O. Ducrot nous semble la plus connue aussi nous en reprendrons le schéma global d'analyse, même si les travaux sur la pertinence ont permis d'avancer dans l'étude des connecteurs, surtout en ce qui concerne la participation du contexte dans l'interprétation du sens. Nous nous permettrons donc d'en aborder quelques aspects (7).

\section{Les connecteurs dans la pragmatique intégrée}

\subsection{Fonctionnement général}

Les connecteurs en langage naturel - leur usage ne suppose pas de conditions de vérité comme pour les connecteurs logiques - s'analysent, non isolément, mais dans des structures sémantiques du type $P$ connecteur $Q$. Ils établissent un lien entre deux entités sémantiques, $P$ et $Q$, qui ne correspondent pas nécessairement à des propositions textuelles: "les premiers [connecteurs logiques] relient des segments toujours présents et identifiables, alors que les seconds [connecteurs naturels] ne concernent pas des segments matériels, mais des contenus sémantiques ou des actes de parole. " (O. Ducrot, Les mots du discours, 1980: 182). Les connecteurs sont donc liés de façon privilégiée à l'implicite et il s'agit pour le destinataire d'identifier les propositions $P$ et $Q$ pour interpréter l'énoncé ( $P$ et $Q$ n'étant pas toujours les éléments linguistiques encadrant le connecteur). Le destinataire est toujours sollicité de "lire " activement un connecteur pour ne pas laisser échapper la conclusion qu'il doit tirer de ce que dit le locuteur.

\subsection{Critique}

La description des connecteurs appartient au cadre théorique général de la pragmatique intégrée. La séquence $P$ connecteur $Q$ est décrite dans le schéma sémantique général proposé au préalable (voir Le dire et le dit, 1984): les expressions linguistiques prises en compte sont donc les phrases (entités syntactico-sémantiques abstraites) et non les énoncés (entités pragmatiques réelles). II s'agit de dégager la signification des phrases et non seulement de déterminer le sens des énoncés. Nous sommes confrontés ici au

5. "ensemble des savoirs qu'un sujet parlant possède sur le fonctionnement de ces "principes" discursifs qui sans être impératifs au même titre que les règles de bonne formation syntactico-sémantique, doivent être observés par qui veut jouer honnêtement le jeu de l'échange verbal ", C. KerbratOrecchioni, 1986: 194.

6 . Voir la présentation des théories sur les connecteurs, dans $\mathrm{J}-\mathrm{M}$. Luscher, Langage et pertinence: 175-227.

7. Pour une présentation de la théorie de la pertinence, voir l'introduction à Langage et pertinence, 1994. problème du choix du « discours idéal » pour l'analyse, celui qui ne s'observe pas mais qui se construit.

De plus, l'approche dérivationnelle (on "dérive", à partir d'instructions - sémantiques - inscrites dans la signification des phrases, les valeurs des variables $P$ et $Q$, qui ont une valeur de base et dont on spécifie les valeurs ajoutées $d u$ fait de la spécificité de la situation de discours, pour chaque emploi du connecteur) que constitue la théorie de Ducrot, s'attache aux propriétés des entités reliées et les différents emplois du connecteur sont justifiés par les différences de nature de ces entités. Ainsi, l'étude est trop souvent la qualification des différentes actualisations de $P$ et de $Q$ plutôt que l'étude des caractéristiques intrinsèques du connecteur. Par exemple, le connecteur peut enchaîner sur un contenu propositionnel $P$, sur un acte illocutoire $F(P)$, sur l'acte d'énonciation $E(P)$.

Néanmoins, cette analyse a fondé la notion moderne d'argumentation (8), en posant que les mécanismes argumentatifs dépendaient autant - si ce n'est plus - de la structure linguistique des énoncés que de leur seule teneur informative.

\subsection{Fonctionnement de mais}

Cette conjonction n'introduit pas d'opposition entre deux segments, un qui précéderait mais, l'autre qui le suivrait, mais les phrases indiquent que "le locuteur voit une opposition entre des entités sémantiques liées à ce qui précède et à ce qui suit mais. Mais les phrases ne disent pas quelles sont ces entités: elles prescrivent simplement à l'interprétant de chercher, vue la situation de discours, entre quelles entités sémantiques liées à ces segments le locuteur établit une relation d'opposition. " (Ducrot et al., 1980: 16). A partir de ces considérations, on peut formuler ainsi le schéma général de fonctionnement de cette conjonction dans la séquence $P$ mais $Q$ : le locuteur $L$ présente $P$ comme un argument en faveur d'une conclusion $R$ (comme devant amener l'interlocuteur à conclure $\mathrm{R}$ ) et dans un deuxième temps il présente $Q$ comme un contre-argument en faveur de non-R, $Q$ étant plus fort pour non-R que $P$ pour R(9). Mais oppose deux informations qui ne sont pas nécessairement opposées en elles-mêmes (c'est-à-dire contradictoires en logique) mais dont les mouvements argumentatifs respectifs sont opposés. Mais marque surtout le rapport que le locuteur entretient avec la situation de communication (autant

8. L'Argumentation dans la langue, Liège, Mardaga, 1983, avec J.-C. Anscombre.

9. Sur la " force" de $Q$ par rapport à $P$, Ducrot écrit dans un article publié plus tard: « En fait, la seule chose constante, c'est que le locuteur déclare négliger le premier [énoncé] dans l'argumentation qu'il est en train de construire et s'appuyer seulement sur le second - la force argumentative supérieure accordée à celui-ci n'étant qu'une justification de cette décision ", in "Opérateurs argumentatifs et visée argumentative „, Cahiers de linguistique française, $n^{\circ} 5$ (1983: 9).

On voit alors tout l'intérêt pragmatique que peut avoir un locuteur de mentionner - par un phénomène de polyphonie, très présent dans les mécanismes argumentatifs - un argument qu'il ne fait pas sien mais qu'il sait être valide dans l'esprit de son interlocuteur dans le seul but de le dévaloriser auprès de celui-ci. Cette technique est omniprésente dans les interactions chez Crébillon. 
les relations avec l'interlocuteur que les données verbales et extra-verbales); plusieurs caractéristiques doivent donc être prises en compte et décrites pour analyser le lien qu'établit le connecteur entre des entités sémantiques, déterminer la visée argumentative de l'énoncé et les nouveaux rapports qui se sont instaurés entre les interlocuteurs:

- le contexte explicite de l'énoncé (situation d'énonciation et compétences respectives des locuteurs)

- les intentions des locuteurs

- les jugements implicites que les interlocuteurs se font sur la situation de communication

- les attitudes qu'ils s'attribuent les uns par rapport aux autres par rapport à cette situation.

Pour décrire entièrement le fonctionnement de mais, il faut faire appel à la notion de topos qu'a développée Ducrot et qui correspond à la notion d'assomption contextuelle chez Luscher. Souvent, pour interpréter l'énoncé contenant mais, on a besoin de "formuler " une prémisse implicitée $P$ ' associée à $P$ et que Ducrot appelle topos. Soit l'exemple :

(1) Cette fille est belle mais intelligente.

Dans (1), $P^{\prime}=$ en général, une belle fille est sotte. $P$ ' fait partie de l'univers de croyance du locuteur et donne une information supplémentaire à l'interlocuteur sur l'interprète. Or, comme le souligne Luscher (1994: 218), il se peut que cette " implicitation provoque l'essentiel des effets obtenus par l'interprétation de cet énoncé " et ne soit pas seulement une exploitation d'une particularité du connecteur mais. Se pose alors la question de l'intention argumentative du locuteur d'une part (peu prise en compte dans la théorie de la pertinence) c'est-à-dire la question de savoir si le locuteur veut poser la prémisse $P$ ' comme la principale « information » qu'il livre à l'interlocuteur ou si cette prémisse est subordonnée, parce qu'admise - ou inconsciemment mise en œuvre dans le cadre éthique, idéologique... du locuteur à la conclusion qu'il veut que l'interlocuteur tire de son énoncé $P$ mais $Q$. Dans ce cas, il " appartient " à l'interprète de considérer la prémisse comme tellement frappante que son effet contextuel constitue l'essentiel de l'interprétation qu'il tire de l'énoncé. Comme le souligne Luscher "l'évaluation de la pertinence est du strict ressort de l'interprète et n'est en aucune façon fonction de la plus ou moins grande distance entre l'intention informative et l'interprétation de l'énoncé. " (1994: 219). II n'en reste pas moins que pour l'analyse pragmatique le cas du « ratage intentionnel » du locuteur est pour le moins intéressant, surtout quant aux stratégies de remplacement qu'il va mettre en œuvre dans l'interaction pour récupérer cette " assomption contextuelle " et essayer de la rendre transparente à l'interlocuteur sans jamais la dire (du “pouvoir pragmatique » de l'implicite).

\section{B. PREMIER DIALOGUE DES ÉGAREMENTS (GF, 77-82)}

\section{Engager la conversation (mais 1)}

Ce mais 1 se situe dans la première intervention de Lursay, qui rompt ainsi le silence gênant qui s'était installé, silence d'autant plus gênant que chacun veut engager la conversation, mais soit timidité, soit respect des bienséances, aucun ne se "lance». Enfin la Marquise se décide: quoi de mieux pour ouvrir un dialogue que de proposer un sujet sur lequel débattre? Le mais sert donc à proposer un énoncé contenant le germe d'une discussion possible, par la présentation de deux propositions aux orientations argumentatives opposées. II se situe à l'intérieur d'une réplique, on peut l'interpréter de la façon suivante: $P$ (l'intrigue n'en est pas neuve) pourrait vous amener à conclure que la pièce était ratée et que cela ne m'a pas intéressée (en vertu du topos qu'il faut de l'originalité pour intéresser), or il n'en est rien parce que $Q$ (j'en ai aimé les détails), et $Q$ est plus fort pour non-R que $P$ pour $R$. Ce propos suggère sans ambiguitté à Meilcour qu'il lui faut répondre, Madame de Lursay lui demande même explicitement ce qu'il en pense, peut-être par peur de le voir rester muet. Sans prétendre argumenter en faveur d'une conclusion à thèse, ce mais, à caractère pragmatique, est intéressant par rapport au mais suivant, celui de la réplique de Meilcour.

\section{Réticences déplacées et offensantes (mais 2)}

Meilcour - seule son inexpérience peut l'absoudre - ne répond pas à la question de la Marquise mais à ce qu'implique la question par rapport à ses compétences, quasi nulles sur le sujet : il s'avoue ignorant en matière de théâtre. Mais au-delà de cet aveu d'ignorance qui ne concerne que lui-même, il refuse de se prêter aux prétentions de l'acte illocutoire. Tout acte de langage est compris comme ayant des prétentions : celles d'être légitime (c'est-à-dire d'avoir le droit d'être accompli, d'être autorisé), et celles de modifier la situation juridique des interlocuteurs, par la création de droits et de devoirs. Une question demande ainsi à recevoir une réponse: ici, Meilcour s'oppose aux prétentions de l'acte de langage de la Marquise, et l'offense "conversationnellement " en refusant de répondre à cette question (il ne sait pas encore que dans le monde il ne faut pas être connaisseur pour bien et longuement parler des choses). Or, en toute bonne foi de profane, il ne veut que réduire la prétention de son acte de langage à venir (son assertion contenant mais) : il va parler de la pièce mais ne prétend pas orienter la Marquise vers la conclusion qu'il est connaisseur(10). Le jeune Meilcour a donc répondu sur le contenu littéral des propos de la Marquise et n'a pas reconnu le caractère d'illocutoire dérivé de sa question: elle ne valait pas tant par sa demande d'information à propos de l'opinion du jeune homme que par sa valeur de questionnement et d'engagement de la conversation.

10. Ce détour est très fréquent dans une relation hiérarchique où le locuteur se sent ou veut se montrer « inférieur » à son interlocuteur: il s'agit de dévaloriser son propos en en réduisant les prétentions illocutoires (dire quelque chose même si on n'est pas le plus autorisé, et même si on ne prétend pas modifier les croyances de l'interlocuteur). Ce détour fonctionne d'autant mieux par antiphrase, dans le cas de la fausse modestie. 
Le connecteur concessif dans l'énoncé de Meilcour va opérer l'inverse de ce qu'avait fait mais dans l'énoncé précédent de Madame de Lursay et presque "ruiner " le débat. Madame de Lursay avait opposé "l'intrigue" (dont on ne pouvait rien dire d'intéressant - parce que banale) aux " détails" (originaux et dont on peut donc parlen). Elle engage Meilcour à lui parler des détails, et ainsi à s'émouvoir de la déclaration d'amour, seul point important, et dont la pièce n'a été que le prétexte discursif. Or Meilcour oppose inversement le " général " (qui correspond à l'“ intrigue") aux "beautés" et "défauts" (les "détails"); il poursuit dans sa logique de profane: en général, elle m'a plu vous pourriez en conclure que je suis connaisseur, il n'en est rien car je ne peux vous parler de ces détails.

L'argumentation en ce début de conversation vaut moins par ia force des arguments et des conclusions à tirer (ce ne sera pas le cas par la suite) que par le décalage manifeste des deux interlocuteurs dans l'échange verbal qui se noue. Madame de Lursay et Meilcour ne sont pas sur la même longueur d'onde: Meilcour croit que Lursay attend de lui un discours critique, et que la discussion doit s'installer au niveau du contenu et des idées, alors qu'elle attend de lui qu'il n'en reprenne que la dynamique interactive et qu'il lui parle. Ce qui manque à Meilcour, c'est bien un topos de la société mondaine, formulé par Versac plus loin:

" c'est qu'en général vous ne pouvez assez vous emparer [de la conversation]. L'essentiel dans le monde n'est pas d'attendre pour parler que l'imagination fournisse des idées. (...)

J'ai vu beaucoup de ces gens stériles, qui ne pensent, ni ne raisonnent jamais, (...) mais qui parlent avec un air de capacité des choses mêmes qu'ils connaissent le moins, (...) l'emporter sur des gens de beaucoup d'esprit, qui, modestes, naturels et vrais, méprisent également le mensonge et le jargon. " (216)

Ces deux mais fonctionnent par rapport aux «stratégies discursives » des deux personnages: l'une veut orienter la conversation et la maintenir sur le terrain de l'amour, l'autre commet quelques bévues conversationnelles qui gênent le déroulement de l'interaction.

\section{Argumentation et dévoilement (mais 3)}

Mais ici réfute l'orientation argumentative prise par "sans doute" qui implique l'acquiescement de la Marquise à la théorie de Meilcour, et qui en reconnaît par là le bien-fondé : le jeune homme pense qu'une femme n'a aucune crainte à avoir à se déclarer et certainement pas celle d'essuyer un refus d'un homme ou celle de prendre le risque d'être moins aimée. Du point de vue de la Marquise, se déclarer, pour une femme, met en danger sa face narcissique et l'expose à un dangereux affadissement de l'amour naissant, selon le topos en usage : une femme a d'autant plus de prix qu'elle a opposé une résistance - même factice - d'autant plus grande au désir de conquête de l'homme. Là aussi, l'argumentation révèle des topoï inconnus à Meilcour et que la Marquise doit tenir encore secrets pour ne pas apparaitre libertine aux yeux candides de Meilcour. Ce mais est donc concessif : la conclusion qu'on peut tirer de $\mathrm{P}$ ("sans doute") est bien " je suis d'accord avec vous", et le connecteur introduit une proposition $Q$ qui contredit cette conclusion sans pour autant contester la validité générale de $P$. En fait Lursay admet que Meilcour a raison dans un certain univers de croyance et introduit par la concession l'existence d'un autre univers auquel elle veut faire accéder Meilcour, et ce en douceur.

Ce mais illustre parfaitement le mécanisme particulier de la conviction sans persuasion: Lursay paraît convaincue et adhérer au raisonnement de Meilcour (mais de façon circonspecte: "sans doute ") sans en être pour autant persuadée : elle ne peut pas adhérer jusqu'au bout à la théorie du jeune homme et ainsi passer à l'acte, dans la mesure où certains topoï entrent en contradiction avec le raisonnement de Meilcour. Sa morale à elle est utilitariste (elle parle en termes d'، intérêt "), elle est donc obligée pour se sortir d'une argumentation dangereuse d'user d'un argument ad hominem ("Vous-même qui vous récriez actuellement contre l'injustice des hommes, vous agiriez comme eux si une femme prévenait vos soupirs") alors que jusqu'à maintenant le débat se situait au niveau "des femmes" et "des hommes", pour attaquer personnellement Meilcour et l'obliger à se défendre plutôt que d'inférer à partir de ce qu'elle vient d'admettre ce qu'elle veut encore lui cacher.

\section{4. Énonciation et séduction}

\section{1. mais 4}

Le connecteur, en tête de réplique, enchaîne sur les propos de Lursay. La séquence $P$ mais $Q$ ne figure pas telle quelle et il faut identifier $P$ par rapport à la réplique de Lursay. Le mais joue à deux niveaux: le dit et le dire. Visiblement, Meilcour ne contredit pas la Marquise (il acquiesce même à ce qu'elle vient de dire dans la relative en incise) : l'emploi de mais suppose au contraire un certain type d'accord avec un élément présenté par $P$, qui serait en gros ici l'accord avec l'assertion énoncée par Lursay [la maladresse d'un amant se déclarant séduit plus que l'élégance de la formulation(11)]. L'accord n'est pas explicite, mais implicité par mais, et il faut le penser pour rendre compte de l'opposition qu'il introduit (12). Cet emploi de mais nous paraît intéressant dans la perspective conversationnelle : il permet en effet la relance du dialogue, à partir de l'aveu d'un consensus qui pourrait bloquer l'échange. Dans la perspective de Roulet (et al., 1985) et Moeschler (1985), le connecteur, outre sa fonction argumentative, qualifie le type d'acte représenté par $P$ et par $Q$, et introduit un élément de relance de la conversation: «Je suis d'accord avec vous $(P)$, vous pour-

riez

11. Versac le dit autrement lors de la leçon de l'Étoile: "(Souvenezvous) qu'en songeant à ce que l'on a à dire, on perd le temps de parler, et que, pour persuader, il faut etourdir." (GF: 216).

12. La théorie des connecteurs, dans le cadre de la théorie de la pertinence, réfute justement cette volonté de maintenir la valeur d'opposition introduite par mais. Voir Luscher, 1994: 175-227. 
penser que je n'ai plus rien à vous dire, or il n'en est rien car $Q=$ je vous pose la question suivante". Mais joue le rôle de marqueur métadiscursif, permettant de relancer la conversation, il ne contredit pas le dit (le contenu référentiel) mais le dire (l'énonciation), c'est-à-dire l'acte de parole accompli par Lursay. Son assertion prétend orienter Meilcour vers la conclusion $R$ ( " vous êtes un jeune amant maladroit, vous avez ainsi plus de force de conviction, donc déclarez-vous "). Meilcour admet l'acte de parole de Lursay, mais en restreint le champ de validité et donc les prétentions en posant sa question ("si la maladresse d'un amant révèle sans aucun doute son amour, elle ne garantit pas la réussite et donc la persuasion $"$ ).

Meilcour révèle ici, d'une part, sa volonté de prolonger la conversation, quels qu'en soient les risques (être notamment obligé de se dévoiler avant d'avoir reçu quelque preuve d'amour susceptible d'éliminer la crainte d'être ridicule et éconduit) et d'autre part, entend bien faire comprendre à la Marquise qu'il ne se prêtera pas à toutes les prétentions de ses actes de paroles. La Marquise est alors obligée de faire une pirouette (79): si l'homme ne persuade pas, on ne lui en tient pas compte (!), et d'user d'un argument d'autorité: "il est plus avantageux, même plus raisonnable, de parler que de s'obstiner à se taire."

Ce mais manifeste deux traits de l'écriture crébillonienne: d'une part, au niveau de l'écriture romanesque, il montre l'art du dialogue, de l'écriture des échanges verbaux faits de points d'arrêt et de relances, et d'autre part, au niveau des paroles échangées, il montre les vicissitudes des mécanismes langagiers de l'argumentation et de la séduction. II s'agit toujours d'imposer ses prétentions, de les faire légitimer par l'autre et ainsi de le faire adhérer à son opinion, pour le faire agir.

\section{2. mais 5}

Nous avons ici la réponse de "la bergère au berger": le mais de Meilcour lui avait servi à endiguer les prétentions de l'acte d'assertion de Lursay au moment où il était acculé à se dire d'accord avec elle et à en tirer les conséquences (cette conclusion allait à l'encontre de ce qu'il défendait, à savoir qu'il est risqué pour un homme d'avouer son amour à une femme). Il avait ainsi réussi à se dégager du filet argumentatif qui se resserrait autour de lui.

C'est maintenant à Lursay de concéder du terrain dans son argumentation et d'accepter le bien-fondé des arguments de Meilcour. Pour contrer cette orientation prise par la conversation, elle s'échappe de la généralité des arguments, et en propose un autre: "vous êtes le seul qui trouviez cela incommode ", argument risqué parce qu'il révèle sa connaissance du monde et des principes de la séduction mondaine ("le seul " vs "tout le reste que je connaisse") et parce qu'il présente d'elle l'image d'une femme peu honnête. Aussi justifie-t-elle son argument (et sa conclusion) par les conséquences d'un tel acte (se déclarer), conséquences que ne nie pas Meilcour, mais qui n'étaient pas le sujet de son argumentation (une déclaration est difficile à faire et peut amener le ridicule sur celui qui s'y risque). Mais, à l'intérieur de cette réplique, revient sur l'acte d'énonciation et introduit un débrayage énonciatif métadiscursif, qui permet un retour sur le discours de Meilcour et l'abandon du sien. Il s'agit là d'un mais presque " auto-dirigé " sur l'énonciation de Lursay, sorte d'appui discursif qui autorise le changement de propos en atténuant l'incongruité du coq-à-l'âne: " Je dis cela, je pourrais aller trop loin dans mes arguments et risquer de me perdre dans et avec mon argumentation, alors que ce qui est important ce n'est pas de persuader Meilcour de la vérité de ce que je dis mais de la persuader de se déclarer à moi, je change donc de sujet ". Ce mais suspend l'orientation argumentative des propos de Lursay, passe au niveau de l'énonciation de Meilcour pour en dévoiler les motivations.

Mais est ici le connecteur de la séduction par excellence: il montre que la séduction vise à persuader l'interlocuteur non d'un faire-croire mais d'un faire-faire. On retrouve ce mécanisme dans beaucoup de scènes de séduction chez Crébillon: l'homme séduit une femme non pas en lui faisant croire qu'il l'aime (il refuse même parfois de le lui dire, ou bien s'il accepte de le dire il le lui fait payer très cher) mais en voulant qu'elle cède. L'argumentation est toujours affaire très serrée chez Crébillon, elle vise à “ impressionner " l'autre (le séducteur le plus noir n'hésite pas à se servir du bluff) en la faisant adhérer à sa propre mesure des choses : argumenter, c'est souvent piéger l'autre en amenant son dispositif argumentatif dans une impasse, et pire, c'est souvent l'amener à adhérer à ses conclusions malgré lui. La conversation en est le champ de bataille, à la fois rituel galant et "honnête", et lieu de séduction sans merci.

Geneviève SALVAN

\section{RÉFÉRENCES BIBLIOGRAPHIQUES}

Pour le texte de Crébillon, nous nous référons à l'édition des Égarements du cœur et de l'esprit, de Jean Dagen, GarnierFlammarion, 1985.

Anscombre J.-C. \& O. Ducrot, L'argumentation dans la langue, Liège, Mardaga, 1983.

Ducrot 0 ., "Opérateurs argumentatifs et visée argumentative ", Cahiers de linguistique française, $n^{\circ} 5,1983$.

- Le dire et le dit, Paris, Hermann, 1984.

Ducrot O. et al., Les mots du discours, Paris, Minuit, 1980. Kerbrat-Orecchioni C., L'implicite, Paris, Armand Colin, 1986.

Maingueneau D., L'Analyse du discours, Introduction aux lectures de l'archive, Paris, Hachette, 1991.

Mœschler J., Argumentation et conversation, Paris, Hatier Credif, 1985.

Roulet et al., L'articulation du discours en français contemporain, Berne, Francfort s/Main, Peter Lang, 1985. 